\title{
The global challenges and opportunities in the practice of rheumatology: White paper by the World Forum on Rheumatic and Musculoskeletal Diseases
}

\author{
Mustafa Al Maini • Femi Adelowo • Jamal Al Saleh • Yousef Al Weshahi • \\ Gerd-Rüdiger Burmester • Maurizio Cutolo • Joseph Flood • Lyn March • \\ Heather McDonald-Blumer • Kevin Pile • Carlos Pineda • Carter Thorne • Tore K. Kvien
}

Received: 14 November 2014 /Revised: 28 November 2014 / Accepted: 1 December 2014 / Published online: 14 December 2014

(C) The Author(s) 2014. This article is published with open access at Springerlink.com

\begin{abstract}
Rheumatic and musculoskeletal diseases (RMDs) represent a multitude of degenerative, inflammatory and autoimmune conditions affecting millions of people worldwide. Persons with these diseases may potentially experience severe chronic pain, joint damage, increasing disability and even death. With an increasingly ageing population, the prevalence and burden of RMDs are predicted to increase, placing greater demands on the global practice of rheumatology and related healthcare budgets. Effective treatment of RMDs currently faces a number of challenges in both the developed and
\end{abstract}

Electronic supplementary material The online version of this article (doi:10.1007/s10067-014-2841-6) contains supplementary material, which is available to authorized users.

\footnotetext{
M. Al Maini

Mafraq Hospital, Abu Dhabi, United Arab Emirates

F. Adelowo

Lagos State University College of Medicine, Ikeja, Nigeria

J. Al Saleh

Dubai Hospital, Dubai, United Arab Emirates

\section{Y. Al Weshahi}

Oman Medical Specialty Board, Muscat, Sultanate of Oman

G.-R. Burmester

Department of Rheumatology and Clinical Immunology, Charité University Medicine Berlin,

Humboldt University, Berlin, Germany

\section{Cutolo}

Division of Rheumatology, Department of Internal Medicine, University of Genova, Genova, Italy

J. Flood

Ohio State University College of Medicine and

Public Health and Columbus Arthritis Center,

Columbus, OH, USA
}

developing world, and individual countries may face more specific local challenges. However, limited understanding of the burden of RMDs amongst public health professionals and policy-makers means that these diseases are often not considered a public health priority. The objective of this review is to increase awareness of the RMDs and to identify opportunities to address RMD challenges on both a local and global scale. On 26 September 2014, rheumatology experts from five different continents met at the World Forum on Rheumatic and Musculoskeletal Diseases (WFRMD) to discuss and identify
L. March

University of Sydney Institute of Bone and Joint Research and Department of Rheumatology, Royal North Shore Hospital, St Leonards, Australia

H. McDonald-Blumer

Department of Medicine, University of Toronto, Toronto, Canada

K. Pile

University of Western Sydney, Sydney, NSW, Australia

C. Pineda

Instituto Nacional de Rehabilitación, Mexico City, Mexico

C. Thorne

University of Toronto and Southlake Regional Health Centre,

Newmarket, ON, Canada

T. K. Kvien

Department of Rheumatology, Diakonhjemmet Hospital, Oslo, Norway

M. Al Maini $(\bowtie)$

WFRMD, PO BOX 77893, Abu Dhabi, United Arab Emirates e-mail: mmaini@wfrmd.org 
some key challenges for the RMDs community today. The outcomes are presented in this review, focusing on access to rheumatology services, diagnostics and therapies, rheumatology education and training and on clinical trials, as well as investigator-initiated and epidemiological research. The longterm vision of the WFRMD is to increase perception of the RMDs as a major burden to society and to explore potential opportunities to improve global and local RMD care.

Keywords Clinical trials · Diagnostic tests · Epidemiology · Medical education $\cdot$ Public health $\cdot$ Rheumatic diseases

\section{Introduction}

The term rheumatic and musculoskeletal diseases (RMDs) largely encompasses over one hundred degenerative, inflammatory and auto-immune conditions which in their most advanced form are associated with severe pain, joint damage, disability and even death. In the 2010 World Health Organization (WHO) Global Burden of Disease Study, RMDs were reported to be the second leading cause of disability worldwide, as measured by years lived with disability [1]. Estimates suggest that almost 2 billion people are affected worldwide [1] imposing huge financial costs; in Europe alone, RMDs are associated with an economic burden of over $€ 200 \mathrm{bn}$ per year. The global burden of individual RMDs has recently been addressed in a series of individual articles (Fig. 1) [2-7]. Nevertheless, awareness of the burden of RMDs amongst policy-makers remains limited for both paediatric and adult manifestations of diseases. With an ageing global population, the prevalence and burden of RMDs in developing and developed countries ${ }^{1}$ is predicted to increase, resulting in reduced quality of life and loss of work productivity, while placing a major burden on national healthcare systems $[8,7]$.

Worldwide inequalities exist in access to clinical care, rheumatology training and research opportunities. However, the burden of disease is often higher in developing countries, due to limited access to clinical services and treatments [9, 10]. Increasing awareness amongst policy-makers of the health problems and economic burdens associated with RMDs, in order to prioritise the RMDs in healthcare planning, will help ensure the best possible patient outcomes. Over the past decade, the WHO has developed a global strategy for the treatment of many non-communicable diseases; however, RMDs are not mentioned [11] and are not indexed as a topic on the WHO website. Other initiatives such as the Bone and Joint Decade (BJD) [11] and the European League Against

\footnotetext{
${ }^{1}$ For the purpose of this manuscript, developing countries are defined by World Bank criteria as low- and middle-income economies, i.e. those with gross national income per capita calculated using the World Bank Atlas method, of \$1045-12,746 in 2013.
}

Rheumatism (EULAR) have advocated for priority to be given to RMDs at the policy level, to reflect the significant challenges these conditions pose to public health. This review identifies some of the main challenges and opportunities for the RMDs community today (Fig. 2).

\section{Methodology}

A preliminary PubMed literature search focusing on challenges in the areas of RMDs clinical care, education and research was conducted to identify key questions for the authors to address. Subsequently, a pre-agreed agenda was discussed at the inaugural meeting of the World Forum on Rheumatic and Musculoskeletal Diseases (WFRMD), held in Abu Dhabi on 26 September 2014. A more focused literature search was then conducted on the key topics identified, with further discussions between authors during development of the final manuscript.

\section{Challenges within the clinical care services}

According to the WHO, a key component of a wellfunctioning health system is to provide equitable access to people-centred care [12]. Availability of healthcare workers, clinical services, affordability of care and cultural acceptability of treatment are all important factors [13]. Any disparity and inadequacy in patient access to healthcare professionals, including primary care providers $\left(\mathrm{PCPs}^{2}\right)$ rheumatologists, orthopaedic surgeons, physical medicine and rehabilitation specialists, nurses, occupational therapists and physiotherapists can significantly delay diagnosis of RMDs and treatment initiation, both of which are key to minimising disease progression and improving patient outcomes [14, 15].

\section{Access to rheumatology services}

Although the number of rheumatologists in developed countries far exceeds that in developing countries, there are worldwide (Table 1) and regional shortfalls in the provision of rheumatologists. A Markov prediction model examining supply and demand for rheumatologists in the United States (US) found that the situation is likely to worsen over the coming decades [16], largely due to an ageing population. Increasing rates of non-communicable diseases, on top of existing endemic and emerging diseases, are also likely to compete for limited resources [17].

\footnotetext{
${ }^{2}$ PCPs are healthcare practitioners who provide primary care for patients, defined by the WHO as first-contact, accessible, continued, comprehensive care, which coordinates with other specialists to provide any other care the patient may need.
} 
Fig. 1 Rheumatic and musculoskeletal diseases (RMDs) and their estimated prevalence
RMD

Low back pain

Other RMDs

Neck pain

Osteoporosis

Osteoarthritis (Hip)

Osteoarthritis (Knee)

Rheumatoid arthritis

Gout

Other RMDs included:

- Ankylosing spondylitis

- Arthralgia

- Behcet's disease

- Carpal tunnel syndrome

- Epicondylitis

- Fibromyalgia

- Myofascial pain syndrome
Prevalence(UI)

$9.4 \%(9.0-9.8)^{4}$

$8.4 \%(8.1-8.6)^{7}$

$4.9 \%(4.6-5.3)^{5}$

Not available

$0.85 \%(0.74-1.02)^{3}$

$3.8 \%(3.6-4.1)^{3}$

$0.24 \%(0.23-0.25)^{2}$

$0.08 \%(0.07-0.08)^{6}$
Estimated no. of persons

632 million $^{1}$

561 million $^{1}$

332 million $^{1}$

$\sim 200$ million (women) ${ }^{\mathrm{a}}$

$\sim 60$ million $^{b}$

251 million $^{1}$

$\sim 17$ million $^{b}$

$\sim 6$ million ${ }^{b}$

Prevalence data derived from 2010 Global Burden of Disease Study, apart from osteoporosis which was not considered a disease in the study. ${ }^{a}$ Estimate from International Osteoporosis Foundation ${ }^{\circ}$ Estimated number of persons derived by multiplying UN estimated global population in $2012(7,162,119,000)$ by prevalence. Ul:

Uncertainty Interval

Analysis of the distribution of rheumatology practices across the US using the 2010 American College of Rheumatology (ACR) membership database found that of 3920 practising rheumatologists, $90 \%$ worked in metropolitan regions, with only $3 \%$ practising in micropolitan areas (populations $<50,000$ ) and $7 \%$ practising in rural areas [18].

\section{Clinical Services}

- Limited access to rheumatology services despite increasing prevalence of RMDs

- Few rheumatologists, particularly in lower socioeconomic areas and developing countries

- Variable availability and trainingin diagnostic techniques

- Access to therapies very dependent on health system and funding

\section{Education}

- Limited coverage of RMDs throughout the medical education continuum

- Lack of knowledge of RMDs amongst general healthcare practitioners and patients

- Restricted opportunities for CME in developing countries

- Financial/language barriers to attending congresses
- Psoriatic arthritis

- Scoliosis

Seronegative spondyloarthropathies

- Soft tissue rheumatism

- Systemic lupus erythematosus

- Tendinitis

- + many other unclassified RMD conditions

Increase awareness of RMDs at the policy-maker level

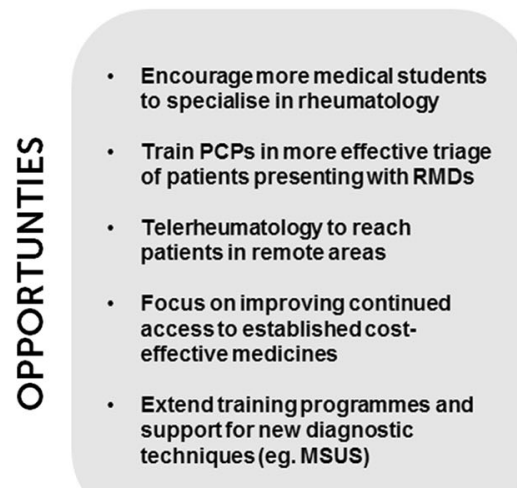

- Liaise with universities to establish and disseminate curricular goals in RMD education

- Encourage student rotations in rheumatology, provide sponsored fellowships and sabbaticals

- Facilitate dissemination of congress talks and workshop materials online

- Reduced fees for rheumatologists from developing countries to attend congresses

- Further campaigns to educate and raise awareness about RMDs

\section{Research}

- Majority of clinical trials take place in Western industrialised countries, RCT data for different subpopulations is very limited

- Lack of support and time for investigator-led research

- Lack of epidemiological data and registries in developing countries causes difficulties in estimating burden and prevalence of RMDs
Facilitate the conduct of more rheumatology research, especially investigator-led research

- Encourage dialogue between FDA/EMA and people coordinating clinical trials

- Provide more discussion groups on how to conduct clinical trials and identify potential issues for RCTs in developing countries

- Encourage development of pragmatic studies, including collection of comparable local registry data

Fig. 2 Summary of the global challenges and opportunities facing rheumatology today 
Similarly, in Canada and the Latin American and Caribbean region, rheumatologists are distributed mainly in large cities, leaving micropolitan and rural areas underserved. Findings from two studies in Ontario reported fewer rheumatologist visits for arthritis and inflammatory arthritis patients who lived in less populated areas with lower socioeconomic status $[19,20]$. As a consequence of the worldwide shortage of rheumatologists, RMD patients are more likely to receive attention from a PCP, who may have no formal training in rheumatology [21, 22].

While shortages are documented in rheumatology care in general, paediatric rheumatology care is severely restricted. In Africa, there are only two paediatric rheumatologists [23], with an estimated $159-180$ practising paediatric rheumatologists across the Latin American and Caribbean regions, 325 in the US and around 60 paediatric rheumatologists in the UK. In India, reports suggest that $<50 \%$ of the 1.3 million children with juvenile idiopathic arthritis (JIA) are diagnosed during the first year of their illness due to limited paediatric rheumatology care [24].

Pan American League Against Rheumatism (PANLAR) and National Rheumatology Societies in the Latin American and Caribbean Region recognise that the number of rheumatologists is insufficient to meet demands [17, 25]. The use of geospatial analysis could aid understanding of the access to healthcare and distribution of the RMDs in any predetermined
Table 1 Estimated rheumatologist workforce across different countries

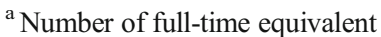
rheumatology physicians, May 2011

${ }^{\mathrm{b}} \mathrm{Number}$ of practising rheumatology physicians per 100,000, May 2011

${ }^{\mathrm{c}} \mathrm{Number}$ of practising rheumatology specialists, 2010

${ }^{d}$ Number of rheumatology physicians (with specialist certificate), May 2011

${ }^{\mathrm{e}}$ Data on file, Italian Society for Rheumatology

${ }^{\mathrm{f}}$ Data from PANLAR National Workforce Survey, 2012

${ }^{\mathrm{g}}$ Number of rheumatologists estimated based on personal c om munication with rheumatologists. Population data were obtained from World Bank mid-year estimates [70] based on year for which the number of rheumatologists were available; 2013 population data was used for 2014 data as 2014 population estimates were not yet available. Ratios were calculated based on number of rheumatologists divided by population estimates for that year

\begin{tabular}{|c|c|c|c|c|}
\hline Country & Year data available & Population & $\begin{array}{l}\text { Estimated number } \\
\text { of rheumatologists }\end{array}$ & $\begin{array}{l}\text { Ratio (per 100,000 } \\
\text { population) }\end{array}$ \\
\hline \multicolumn{5}{|l|}{ Europe } \\
\hline UK [64] & 2011 & $63,258,918$ & $531^{\mathrm{a}}$ & 0.84 \\
\hline Ireland [65] & 2011 & $4,576,794$ & $\sim 23$ & $0.5^{\mathrm{b}}$ \\
\hline France [66] & 2010 & $65,023,142$ & $2,470^{\mathrm{c}}$ & 3.80 \\
\hline Germany [67] & 2011 & $81,797,673$ & $757^{\mathrm{d}}$ & 0.93 \\
\hline Italy $^{\mathrm{e}}$ & 2011 & $60,782,668$ & 800 & 1.3 \\
\hline \multicolumn{5}{|l|}{ North America } \\
\hline $\mathrm{USA}^{\mathrm{f}}$ & 2012 & $313,873,685$ & 5602 & 1.78 \\
\hline Canada [68] & 2013 & $35,158,304$ & 342 & 0.97 \\
\hline \multicolumn{5}{|l|}{ Middle East } \\
\hline $\mathrm{Oman}^{\mathrm{g}}$ & 2014 & $3,632,444$ & 20 & 0.55 \\
\hline $\mathrm{UAE}^{\mathrm{g}}$ & 2014 & $9,346,129$ & 40 & 0.43 \\
\hline Kuwait $^{\mathrm{g}}$ & 2014 & $3,368,572$ & 30 & 0.89 \\
\hline Qatar ${ }^{\mathrm{g}}$ & 2014 & $2,168,673$ & 12 & 0.55 \\
\hline Saudi Arabia ${ }^{\mathrm{g}}$ & 2014 & $28,828,870$ & 120 & 0.42 \\
\hline Bahrain $^{\mathrm{g}}$ & 2014 & $1,332,171$ & 4 & 0.30 \\
\hline \multicolumn{5}{|l|}{ Latin America } \\
\hline Uruguay $^{\mathrm{f}}$ & 2012 & $3,395,253$ & 105 & 3.09 \\
\hline Brazil $^{\mathrm{f}}$ & 2012 & $198,656,019$ & 1543 & 0.78 \\
\hline Colombia $^{\mathrm{f}}$ & 2012 & $47,704,427$ & 136 & 0.29 \\
\hline Nicaragua ${ }^{\mathrm{f}}$ & 2012 & $5,991,733$ & 4 & 0.07 \\
\hline Mexico $^{f}$ & 2012 & $120,847,477$ & 568 & 0.47 \\
\hline \multicolumn{5}{|l|}{ Asia } \\
\hline China [69] & 2007 & $1,317,885,000$ & 2216 & 0.17 \\
\hline India $^{\mathrm{g}}$ & 2014 & $1,252,139,596$ & $\sim 200$ & 0.02 \\
\hline Pakistan [22] & 2014 & $182,142,594$ & 20 & 0.01 \\
\hline Thailand [42] & 2014 & $67,010,502$ & 150 & 0.22 \\
\hline \multicolumn{5}{|l|}{ Africa } \\
\hline Comoros $^{\mathrm{g}}$ & 2014 & 734,917 & 0 & 0 \\
\hline Nigeria $^{\mathrm{g}}$ & 2014 & $173,615,345$ & 22 & 0.01 \\
\hline Djibouti $^{\mathrm{g}}$ & 2014 & 872,932 & 0 & 0 \\
\hline \multicolumn{5}{|l|}{ Australia } \\
\hline Australia $^{\mathrm{g}}$ & 2014 & $23,130,900$ & 307 & 1.33 \\
\hline
\end{tabular}


geographic area [26]. A study of US Medicare patients found that increased driving distance to rheumatologists was associated with decreased odds of diagnosis with rheumatoid arthritis (RA) [27]. Providing up-to-date information about the local supply of rheumatologists, and additional funds for training programmes, could attract new rheumatologists to underserved regions through migration and expansion [18]. Nevertheless, in some countries, it may never be possible to train a sufficient number of rheumatologists. As such, PCPs may need to play a greater role in early rheumatological care and provide a more effective triage system. The early arthritis clinic model is gaining popularity, in which PCPs are trained to screen patients with RMDs and triage the most severe and urgent cases, such as primary inflammatory arthropathies [28]. The use of telephone and videoconference consultations may provide an effective means to improve access to rheumatology care in rural areas. This would allow for both direct patient care by rheumatologists and support of PCPs who can be educated, mentored and given diagnostic and management advice [9, 29-32].

\section{Access to diagnostics}

Early diagnosis and treatment of RMDs in the "window of opportunity' following the onset of symptoms are crucial to avoid long-term complications but requires specialist knowledge [33]. When appropriate treatment is started early, medical costs, disability and work limitations can be reduced [33].

Currently, there is no single preferred method of diagnosing RMDs, and tools used are dependent on the PCPs' own knowledge [34]. Clinical diagnoses are typically supported by blood tests (e.g. erythrocyte sedimentation rate, C-reactive protein, anti-nuclear antibodies, rheumatoid factor) and imaging modalities. However, lack of expertise or funding and limited imaging facilities can create barriers to more systematic use of magnetic resonance imaging (MRI) and ultrasound in some regions, including Latin America [35]. Use of dualenergy X-ray absorptiometry (DXA) to measure bone mineral density is the standard diagnostic technique for osteoporosis but costs are relatively high, with limited access to equipment in many developing countries [36]. Absolute fracture risk calculators such as the FRAX model (a validated web-based algorithm) may provide a suitable alternative [37]. Common serological tests such as rheumatoid factor and anti-nuclear antibodies are available in all African countries, but rural areas invariably do not have such facilities. In resource-poor settings, with limited access to diagnostics, the assessment of disease activity using clinical rather than laboratory measures could be advantageous (e.g. the modified systemic lupus erythematosus [SLE] Disease Activity Index [SLEDAI]) [38].

Further development of rapid blood or serum-based diagnostic tests to screen for auto-immune RMDs should be encouraged. Point-of-care tests for early detection of RA using auto-antibodies or rheumatoid factor require only a single drop of blood and can be performed within minutes [39]. These advanced and non-invasive diagnostic tools represent an expanding area of interest for rheumatologists. Use of capillaroscopy to examine microcirculatory impairment is also gaining popularity amongst Western and Eastern countries, with dedicated study groups at ACR and EULAR congresses.

Musculoskeletal ultrasound (MSUS) is playing an increasingly important role in optimising clinical assessment of patients with RMDs and substantially improves therapeutic and diagnostic capabilities [40]. However, training is expensive, labour intensive and the learning curve is very steep [41], with huge variation between countries signifying the need for an international consensus on MSUS training [41]. ACR, EULAR and PANLAR have all developed guidelines and international training programmes for MSUS.

\section{Access to therapies}

Access to suitable therapies is largely dependent on the nation's health system, drug availability and the economic status of the country. In wealthy countries, there may be easy access to therapies through government-supported funding or insurance, whereas in poorer countries, patients are offered treatment solely based on their ability to pay for it. In Thailand, patients in the Universal Coverage Scheme can only access medicine on the National List of Essential Medicines, which does not include biologics, and treatment regimens are modified according to the level of patient health insurance cover [42]. Even in the US, patients with insurance coverage may be forced by the insurer to pay high co-payments or "co-insurance". These additional payments may place vital treatment out of the patient's grasp. The ACR spends great time and resources to combat these onerous practices in cooperation with its advocacy partner, the Arthritis Foundation. In Africa, immunosuppressants for the treatment of SLE are unaffordable for the majority of patients, or even state healthcare budgets [38]. Such variable access to therapies can deny implementation of established RMD treatment guidelines in developing countries, such as EULAR recommendations on the use of biologics in RA [43].

In 2011, a cross-sectional study across 46 European countries found that patients with RA in lower income countries had reduced access to both biologic and synthetic diseasemodifying anti-rheumatic drugs (bDMARDs and sDMARDs) [13]. PANLAR has proposed that biosimilars could improve therapy access for patients with RA in the Latin American countries, as could decreasing custom fees and taxes for drugs and joint prostheses [25]. Informal discussions with manufacturers in the US indicates that the cost of biosimilars may not be significantly reduced compared to biologics, with $\leq 20 \%$ price reduction anticipated. However, in the Norwegian tender 
system, the biosimilar Remsima was offered for a price $39 \%$ lower than that for Remicade [44].

Inequities exist in the availability and consistency of supply of established cost-effective medications for RMDs, including non-steroidal anti-inflammatory drugs (NSAIDs; such as ibuprofen, naproxen and diclofenac) and sDMARDs, such as hydroxychloroquine, sulfasalazine, methotrexate (MTX) and leflunomide. In some African countries, disease-modifying anti-rheumatic drugs (DMARDs) are usually stocked by only a few pharmacies. The time taken for patients to receive sDMARDs may also vary depending on the healthcare system; in Brazil, it can take 1-4 years before a patient in the public healthcare system receives a sDMARD, compared with $<2$ years in private healthcare [35]. In South Sudan, DMARDs are not generally available and in other African countries, similar issues exist with SLE medications, alongside the fear of buying counterfeit medications locally [38]. Consequently, patients and their doctors need to obtain medicines from other countries, often at great expense $[45,38]$.

The concept of selling essential medicines in low- and middle-income countries at lower prices than in industrialised countries has received widespread support from industry, policy-makers and academics [46]. However, tiered pricing does not necessarily result in the lowest sustainable prices or lead to price reductions over time [46]. Further research is needed to determine whether a tiered pricing model could successfully increase access to RMD treatments in developing countries.

\section{Education of rheumatologists and other experts in RMD care}

Successful education in the RMDs requires a collaborative, constructive, and contextual approach, aimed at providing education across professions. To address the current challenges in education across the medical education continuum, leadership of RMD experts specialising in medical education, research and service leadership will be mandatory.

\section{Medical school}

Early exposure of medical students, residents and students of other relevant health professions to rheumatology 'culture' is vital to improve awareness of the RMDs and increase the number of students and residents considering this field of study. In many countries, PCPs lack knowledge of the nuances of RMDs, leading to misconceptions about the most appropriate treatment options, delayed diagnoses and a limited awareness of conditions such as the spondylarthropathies.

To date, rheumatology education has not been a high priority in many medical school curricula and consequently does not allow sufficient exposure of students to the RMDs [47]. A 2008 survey of Canadian medical schools found that the total average time spent in musculoskeletal physical examination teaching was just $4-7 \mathrm{~h}$ over 4 years of study, and $58 \%$ of teaching was performed by non-RMD experts [ 48 , 49]. In part, this may be due to the limited number of rheumatologists and other health professionals trained as educators and educational leaders, who can combine their expertise in both RMDs and education to increase focus on RMDs across the education continuum.

\section{Generalist training}

A US survey of PCPs evaluated their experience with the use of DMARDs in RA and found that only a minority initiated this therapy option and PCPs generally had a high level of discomfort prescribing DMARDs [50]. A similar US survey to assess the use of European and American gout treatment recommendations found that of 838 PCP respondents, only half reported optimal treatment practices for the management of acute gout, and $<20 \%$ for intercritical or tophaceous gout, indicating care deficiencies [51]. Typically, PCPs are overextended taking care of other chronic diseases, such as obesity, hypertension and diabetes, which may explain their restricted time devoted to increased understanding of RMDs.

In a survey of undergraduate nursing, occupational therapy and physiotherapy courses in the UK, educationalists reported only limited coverage of rheumatology [52, 53]. However, the ACR's Association of Rheumatology Health Professionals (ARHP) offers a successful programme called Advanced Practice Rheumatology, with a modular online course and hands-on training for advanced practice nurses and physician's assistants. Other societies have similar programmes such as the Rheumatology Nurses Society (RNS), committed to the education of nurses working in rheumatology. In the Latin American countries, a PANLAR-endorsed multinational rheumatology e-learning and presence training diploma programme for nurses and health professionals has been established and includes a hands-on and theoretical MSUS course. Greater provision of online training courses in developing countries, at reduced costs, could further enhance training opportunities.

Paediatric rheumatology is a particular area in which PCPs require further education. A review of the barriers constraining access to appropriate paediatric rheumatology care found that, in general, US paediatricians had little or no training in RMD health and had relatively poor physical examination and diagnosis skills [54]. Introduction of a mandatory RMDs rotation for paediatric residencies could help to improve awareness and understanding of these cases [54]. The ACR currently funds visiting paediatric rheumatology professorships, to bring in-depth training to paediatric programmes 
and provide much needed support for paediatric care in developing countries.

\section{Specialist training}

Collaborations between rheumatology associations and universities could help strengthen existing teaching, provide core knowledge of the RMDs to all practitioners and encourage rheumatology as a specialty within that region. The ACR provides training and research grants for medical students, residents, fellows and clinicians in rheumatology, and has convened an international task force to assess the need for, and distribution of, educational tools. Similarly, the EULAR Committee for Education and Training (ESCET) offers bursaries for students and rheumatologists worldwide to attend online and postgraduate courses on RMDs. The Emerging EULAR Network (EMEUNET) is a working group of young rheumatology clinicians across 77 countries that facilitates education in RMDs. EULAR also offers a subsidised online rheumatology course.

Nevertheless, there is a general lack of training opportunities for those considering specialisation as rheumatologists. For example, in Nigeria, there is only one rheumatology training centre in the country. In Thailand, there are only 15 rheumatology training positions available each year, but this could be improved by increasing the number of grants available from the Thai Ministry of Public Health [42]. Of the current rheumatologists practising in India and Pakistan, 20 and $50 \%$, respectively, received their training elsewhere [22]. In the United Arab Emirates, Bahrain and Oman, all rheumatology training takes place overseas, while in Kuwait, Qatar and Saudi Arabia residents have hybrid programmes of local and international training. Although overseas accreditation can be beneficial, the majority who train in the West do not return to their native countries to practice rheumatology [22].

\section{Continuing education}

Regional and national rheumatology leagues should help to deliver courses with clear curricular goals. The Chinese Rheumatology Association is working with medical schools to provide intensive training sessions, lectures and comprehensive curricula for the purpose of continuing medical education (CME) [55]. The ACR Rheumatology Research Foundation also has programmes for this, plus nascent programmes to attract college students to rheumatology and other rheumatology health professions.

Reduced registration fees or sponsorship to attend international congresses presents an ideal opportunity to promote 'rheumatology without borders' so that physicians worldwide can learn about the latest advances in the treatment of RMDs [56]. The impact of educational forums could be broadened via free post-congress web links that disseminate talks and workshops (using podcasts, videos, slide sets, handouts and translated materials) to target audiences that are unable to attend the congress due to lack of time or funding, travel requirements and language barriers. In addition, world rheumatology leaders could travel to developing regions to share their knowledge and expertise, e.g. the International Advanced Abu Dhabi Rheumatology Review Course (and numerous other meetings around the world).

CME programmes are currently offered by ACR, EULAR, American Board of Medical Specialties (ABMS), Royal College of Physicians (RCP), Asia Pacific League of Associations for Rheumatology (APLAR), PANLAR and African League against Rheumatism (AFLAR). However, there is a general lack of programmes in Africa, Asia and the Middle East. The International League of Associations for Rheumatology (ILAR) Grants Program also provides opportunities to advance the education and clinical practice of rheumatology in developing countries but has limited resources, with grants totalling $\$ 150,000$ in 2014. A successful ILAR-supported project running from 2012 to 2013 addressed a particular need in Zambia to enhance paediatric and adult rheumatology education and practice [57]. Similarly, the ILAR-funded UWEZO Musculoskeletal Health training project aims to provide medics in Kenya with appropriate training to diagnose and treat RMDs (UWEZO means 'capability' in Swahili). Trained medics can then go on to train community health workers in their region.

The Arthritis Alliance of Canada has developed a coordinated national framework for models of care for patients with inflammatory joint diseases. Similarly, the Canadian Rheumatology Association (CRA) is a strong and committed organisation for RMDs, forming alliances with the Canadian Medical Association and the Royal College of Physicians and Surgeons, and increasing relationships with other organisations including ACR and PANLAR.

\section{Patient awareness of RMDs}

In some areas of the world, a lack of basic education and literacy, poorer socioeconomic status and cultural beliefs can influence the decision of patients to seek medical attention from a PCP, or they may only seek help from alternate care practitioners [9]. Many patients see the aches and pains associated with arthritis as a part of life and do not seek medical opinion.

Prevention strategies with campaigns to educate and raise awareness about RMDs may increase the likelihood of a patient approaching their PCP and may also increase a patient's understanding of their disease, ultimately improving their compliance with treatment decisions. The Patient Association inside EULAR (PARE) organises courses and local training sessions for patients across 37 countries. Existing campaigns to educate the public about health issues such as 
diabetes, hypertension and obesity could also be expanded to include RMDs. With the increasing use of smartphones worldwide, patient self-management or education programmes integrated into social media technologies or mobile applications might broaden patient access to information about their disease [58]. Looking ahead, organisations such as the US Arthritis Foundation or the ACR Simple Tasks campaign, which has gained the interest of rheumatology associations worldwide, may play an important role in raising patient and physician awareness and educating policy-makers about the value of rheumatology education [33].

\section{Research challenges}

The impact of financial, political, social, or environmental factors on the opportunity to conduct clinical, epidemiological, as well as basic and translational research creates major challenges worldwide. In many countries, including the US and Canada, government funding for rheumatology research is diminishing and academics are being forced into the clinic, reducing their research time. In countries with major shortfalls in the rheumatology workforce, health systems are likely to need all rheumatologists for full-time clinical practice, leaving insufficient time for research activities.

\section{Clinical trials research}

Ethnicity may influence the manifestation of various RMDs, due to underlying genetic differences, environmental factors, cultures and socioeconomic status [42]. However, pharmaceutical companies running randomised controlled trials (RCTs) have tended to conduct trials in Western industrialised countries, limiting the amount of information for the effectiveness and safety of treatments in non-Western populations (Table 2). Although more trials are now occurring in Eastern Europe and India due to a lack of biologic-naïve patients in the West, the Middle East and North Africa region sponsor less than $1 \%$ of global clinical trials [59].

Action is needed to encourage pharmaceutical companies to conduct RCTs which are more generalisable to different populations. However, a number of challenges must be overcome including training individuals to successfully manage the trials, differences in language, culture, social and health literacy; inaccurate translation and high levels of illiteracy can be problematic [59]. Establishing local Clinical Research Organisations could help overcome regulatory bottlenecks, as they are more likely to understand local regulations and logistic needs [59]. More discussion groups at rheumatology congresses such as ACR, EULAR, AFLAR and PANLAR on how to conduct RCTs may also help to identify potential issues for developing countries. In developed countries,
Table 2 Number of registered clinical trials listed on ClinicalTrials.Gov for different RMDs by region

\begin{tabular}{lccrc}
\hline \multirow{2}{*}{ Region } & \multicolumn{3}{l}{ Number of registered trials } & \\
\cline { 2 - 5 } & $\begin{array}{l}\text { Rheumatoid } \\
\text { arthritis }\end{array}$ & Osteoarthritis & Gout & $\begin{array}{l}\text { Systemic lupus } \\
\text { erythematosus }\end{array}$ \\
\hline Africa & 75 & 24 & 8 & 13 \\
East Asia & 253 & 144 & 13 & 86 \\
Europe & 678 & 530 & 31 & 104 \\
Latin America & 243 & 65 & 9 & 59 \\
Middle East & 89 & 53 & 2 & 16 \\
North America & 693 & 821 & 85 & 238 \\
North Asia & 160 & 12 & 12 & 30 \\
Pacifica & 102 & 43 & 9 & 16 \\
South Asia & 48 & 16 & 2 & 16 \\
Southeast Asia & 61 & 39 & 8 & 24
\end{tabular}

Information based on number of clinical trials registered on ClinicalTrials.Gov by RMD topic, as of 4 November 2014. Studies with no locations are not included; studies with multiple locations are included in each region containing locations

${ }^{a}$ Latin America values obtained by adding number of trials in Central America and South America

increased dialogue is needed between drug regulatory bodies such as the US Food and Drug Administration (FDA) and European Medicines Agency (EMA), pharmaceutical companies and researchers coordinating RCTs. Recently, collaboration between EULAR and the EMA has been officialised, with EULAR members making recommendations together with EMA officers.

Investigator-initiated research

There are many existing programmes to support investigatorinitiated RMDs research including the Canadian Initiative for Outcomes in Rheumatology Care (CIORA) which offers awards to new clinicians to carry out independent arthritis research. Similarly, the ACR Rheumatology Research Foundation funds promising research to help bridge investigators to sustainable support from the National Institutes of Health (NIH). In China, government funding for rheumatology research has increased substantially in the past decade, leading to many high-quality publications and international collaborations [55]. In the recent Arab League Against Rheumatism (ARLAR) meeting, 100 research scholarships were awarded to young rheumatologists. The Paediatric Rheumatology International Trials Organisation (PRINTO), Paediatric Rheumatology European Society (PReS) and Childhood Arthritis and Rheumatology Research Alliance (CARRA) also aim to facilitate and coordinate clinical trials and research in paediatric RMDs. 
Epidemiological research

Real-life efficacy and safety data from registries provide a valuable source of data for the RMDs. For example, the British Society for Rheumatology Biologics Register (BSRBR) has been used to compare the risk of tuberculosis between different biologics used to treat RA [60]. The nascent ACR RISE registry will collect point-of-service data on patients in various settings, with international interest in joining this registry. Similarly, BIOBADAMERICA is a Latin America and Caribbean registry of 15 nations that collects information on all relevant adverse events in patients on biologics [61].

To date, the most comprehensive effort to collect data for the RMDs is the Global Burden of Disease (GBD) 2010 study, which estimated the global burden of RMDs and showed that the prevalence and burden of RMDs are exceptionally high throughout the world [2-7, 62]. However, for many regions, burden estimates had to be derived through predictive modelling [8].

Country-based data is very useful for local clinicians to follow their own patients who may be different in terms of disease severity, access to care, and comorbidities. Such data is also beneficial when negotiating with local governments and payers to provide evidence for the benefit and safety of more expensive treatments. Despite the obvious advantages of collating such information, obtaining the funding to set up new registries can be difficult. Furthermore, academics are not necessarily engaged in this type of activity. In many developing countries, health records are still paper-based; the introduction of electronic records is necessary to maximise data collection and simplify registry management.

The WHO-ILAR Community Oriented Programme in the Rheumatic Diseases (COPCORD) was created to gather data on RMDs, with an emphasis on developing rural economies [63]. The COPCORD Core Questionnaire (CCQ) has provided a useful way of collecting grass-roots data on the prevalence of RMDs in developing countries. However, the methodology is quite labour intensive, and design and methods are limited by stringent budgets, imposing deviations in the core protocol [63]. To harmonise epidemiological data for the RMDs, valid and standardised questions should be developed for use in population-based health interview and examination surveys.

\section{Conclusions}

The high prevalence and burden of RMDs presents a number of global challenges for the practice of rheumatology and more generally for chronic diseases. Issues concerning access to clinical care services, rheumatology education and research are particularly problematic in developing countries and rural areas, yet challenges faced in developed countries hinder the progress of RMD healthcare worldwide. By increasing awareness of the burden of RMDs at the policy-maker level and identifying key challenges, it may be possible to identify realistic opportunities to address the global RMDs problem. The WFRMD aims to address important challenges for the RMDs by facilitating discussions with rheumatology experts and lobbying stakeholders, internal and regional rheumatology associations, local governments and the WHO to raise awareness of these prevalent and burdensome diseases.

Acknowledgments Financial support was provided by the World Forum on Rheumatic and Musculoskeletal Diseases (WFRMD), managed by the K.I.T. Group. Writing support and editorial assistance was provided by Costello Medical Consulting.

Conflict of interest All authors declare no conflict of interest related to this manuscript.

Open Access This article is distributed under the terms of the Creative Commons Attribution License which permits any use, distribution, and reproduction in any medium, provided the original author(s) and the source are credited.

\section{References}

1. Vos T, Flaxman AD, Naghavi M, Lozano R, Michaud C, Ezzati M, Shibuya K, Salomon JA, Abdalla S, Aboyans V (2013) Years lived with disability (YLDs) for 1160 sequelae of 289 diseases and injuries 1990-2010: a systematic analysis for the global burden of disease study 2010. Lancet 380(9859):2163-2196

2. Cross M, Smith E, Hoy D, Carmona L, Wolfe F, Vos T, Williams B, Gabriel S, Lassere M, Johns N (2014) The global burden of rheumatoid arthritis: estimates from the global burden of disease 2010 study. Ann Rheum Dis 73(7):1316-1322

3. Cross M, Smith E, Hoy D, Nolte S, Ackerman I, Fransen M, Bridgett L, Williams S, Guillemin F, Hill CL (2014) The global burden of hip and knee osteoarthritis: estimates from the global burden of disease 2010 study. Ann Rheum Dis 73(7):1323-1330

4. Hoy D, March L, Brooks P, Blyth F, Woolf A, Bain C, Williams G, Smith E, Vos T, Barendregt J (2014) The global burden of low back pain: estimates from the global burden of disease 2010 study. Ann Rheum Dis 73(6):968-974

5. Hoy D, March L, Woolf A, Blyth F, Brooks P, Smith E, Vos T, Barendregt J, Blore J, Murray C, Burstein R, Buchbinder R (2014) The global burden of neck pain: estimates from the global burden of disease 2010 study. Ann Rheum Dis. doi:10.1136/annrheumdis2013-204431

6. Smith E, Hoy D, Cross M, Merriman TR, Vos T, Buchbinder R, Woolf A, March L (2014) The global burden of gout: estimates from the global burden of disease 2010 study. Ann Rheum Dis 73(8): 1470-1476. doi:10.1136/annrheumdis-2013-204647

7. Smith E, Hoy DG, Cross M, Vos T, Naghavi M, Buchbinder R, Woolf AD, March L (2014) The global burden of other musculoskeletal disorders: estimates from the global burden of disease 2010 study. Ann Rheum Dis 73(8):1462-1469 
8. Hoy DG, Smith E, Cross M, Sanchez-Riera L, Blyth FM, Buchbinder R, Woolf AD, Driscoll T, Brooks P, March LM (2014) Reflecting on the global burden of musculoskeletal conditions: lessons learnt from the global burden of disease 2010 study and the next steps forward. Ann. Rheum Dis:annrheumdis-2014-205393

9. Mody GM, Brooks PM (2012) Improving musculoskeletal health: global issues. Best Pract Res Clin Rheumatol 26(2):237-249

10. Massardo L, Pons-Estel BA, Wojdyla D, Cardiel MH, GalarzaMaldonado CM, Sacnun MP, Soriano ER, Laurindo IM, AcevedoVásquez EM, Caballero-Uribe CV (2012) Early rheumatoid arthritis in Latin America: low socioeconomic status related to high disease activity at baseline. Arthritis Care Res 64(8):1135-1143

11. Woolf AD The bone and joint decade: working together to make musculoskeletal conditions a public health priority. http://www. arthritisresearchuk.org/health-professionals-and-students/reports/ topical-reviews/topical-reviews-summer-2012.aspx\#sthash. AvOx0hMb.dpuf. Accessed 5th Nov 2014

12. Key components of a well functioning health system. World Health Organization, 2010. http://www.whoint/healthsystems/EN HSSkeycomponentspdf?ua=1 Accessed 5th Nov 2014

13. Putrik P, Ramiro S, Kvien TK, Sokka T, Pavlova M, Uhlig T, Boonen A, Tafaj A, Harutyunyan R, Radner H (2014) Inequities in access to biologic and synthetic DMARDs across 46 European countries. Ann Rheum Dis 73(1):198-206

14. Fiehn C, Hajjar Y, Mueller K, Waldherr R, Ho A, Andrassy K (2003) Improved clinical outcome of lupus nephritis during the past decade: importance of early diagnosis and treatment. Ann Rheum Dis 62(5): 435-439

15. Nell V, Machold K, Eberl G, Stamm T, Uffmann M, Smolen J (2004) Benefit of very early referral and very early therapy with diseasemodifying anti-rheumatic drugs in patients with early rheumatoid arthritis. Rheumatology 43(7):906-914

16. Deal CL, Hooker R, Harrington T, Birnbaum N, Hogan P, Bouchery E, Klein-Gitelman M, Barr W (2007) The United States rheumatology workforce: supply and demand, 2005-2025. Arthritis Rheum 56 (3):722-729

17. Cardiel MH (2011) Present and future of rheumatic diseases in Latin America. Are we prepared to face them? Reumatología Clínica (Engl Ed) 7(5):279-280

18. FitzGerald JD, Battistone M, Brown CR Jr, Cannella AC, Chakravarty E, Gelber AC, Lozada CJ, Punaro M, Slusher B, Abelson A (2013) Regional distribution of adult rheumatologists. Arthritis Rheuma 65(12):3017-3025

19. Badley EM, Canizares M, Gunz AC, Davis AM (2014) Visits to rheumatologists for arthritis: the role of access to primary care physicians, geographic availability of rheumatologists, and socioeconomic status. Arthritis Care Res

20. Widdifield J, Paterson JM, Bernatsky S, Tu K, Thorne JC, Ivers N, Butt D, Jaakkimainen RL, Gunraj N, Ahluwalia V (2014) Access to rheumatologists among patients with newly diagnosed rheumatoid arthritis in a Canadian universal public healthcare system. BMJ Open 4(1): $\mathrm{e} 003888$

21. Oyoo O, Moots RJ, Ganda B (2012) Stepping into the state of rheumatology in East Africa. Rheumatology:ker411

22. Gibson T (2014) Rheumatology in India and Pakistan today. Rheumatology:keu306

23. Henrickson M (2011) Policy challenges for the pediatric rheumatology workforce: Part III. The international situation. Pediatr Rheumatol Online J 9:26. doi:10.1186/1546-0096-9-26

24. Habibi S, Aggarwal A, Ramanan AV (2012) Paediatric rheumatology in India: challenges and opportunities. Rheumatology 51(6):962-963

25. Cardiel M (2006) First Latin American position paper on the pharmacological treatment of rheumatoid arthritis. Rheumatology 45:ii7ii 22

26. Al-Maini M, Jeyalingam T, Brown P, Lee JJ, Li L, Su J, Gladman DD, Fortin PR (2013) A hot spot for systemic lupus erythematosus, but not for psoriatic arthritis, identified by spatial analysis suggests an interaction between ethnicity and place of residence. Arthritis Rheum 65(6):1579-1585

27. Polinski JM, Brookhart MA, Ayanian JZ, Katz JN, Kim SC, Lii J, Tonner C, Yelin E, Solomon DH (2014) Relationships between driving distance, rheumatoid arthritis diagnosis, and disease-modifying anti-rheumatic drug receipt. Arthritis Care Res

28. Gormley GJ, Steele WK, Gilliland A, Leggett P, Wright GD, Bell AL, Matthews C, Meenagh G, Wylie E, Mulligan R, Stevenson M, O'Reilly D, Taggart AJ (2003) Can diagnostic triage by general practitioners or rheumatology nurses improve the positive predictive value of referrals to early arthritis clinics? Rheumatology (Oxford) 42 (6):763-768. doi:10.1093/rheumatology/keg213

29. Davis P, Howard R, Brockway P (2001) An evaluation of telehealth in the provision of rheumatologic consults to a remote area. $\mathrm{J}$ Rheumatol 28(8):1910-1913

30. Jong M, Kraishi M (2004) A comparative study on the utility of telehealth in the provision of rheumatology services to rural and northern communities. Int J Circumpolar Health 63(4):415-421

31. Leggett P, Graham L, Steele K, Gilliland A, Stevenson M, O’Reilly D, Wootton R, Taggart A (2001) Telerheumatology_diagnostic accuracy and acceptability to patient, specialist, and general practitioner. Br J Gen Pract 51(470):746-748

32. Roberts L, Lamont E, Lim I, Sabesan S, Barrett C (2012) Telerheumatology: an idea whose time has come. Intern Med J 42 (10):1072-1078

33. ACR Simple Tasks Campaign Fact Sheet. http://simpletasks.org/wpcontent/uploads/2013/12/Campaign-Fact_Sheet.pdf. Accessed 5th Nov 2014

34. National Audit Office (NAO) (2009). Services for people with rheumatoid arthritis. Published by the NAO, London, 15 July 2009. http:// www.nao.org.uk/publications/0809/rheumatoid_arthritis.aspx. Accessed 5th Nov 2014

35. Miltenburger C, Munkombwe M, I L A Survey of Barriers to Treatment Access in Rheumatoid Arthritis in Major Latin American Countries-Argentina, Brazil and Mexico. March 2010. http://www.comparatorreports.se/LA\%20RA\%20barrier\%20report FINAL.pdf. Accessed 5th Nov 2014

36. Kruger MC, Todd JM, Schollum LM, Kuhn-Sherlock B, McLean DW, Wylie K (2013) Bone health comparison in seven Asian countries using calcaneal ultrasound. BMC Musculoskelet Disord 14(1):81

37. Kanis JA, Johnell O, Oden A, Johansson H, McCloskey E (2008) FRAX and the assessment of fracture probability in men and women from the UK. Osteoporos Int 19(4):385-397. doi:10.1007/s00198-007-0543-5

38. Tiffin N, Hodkinson B, Okpechi I (2013) Lupus in Africa: can we dispel the myths and face the challenges? Lupus:0961203313509296

39. Egerer K, Feist E, Burmester G-R (2009) The serological diagnosis of rheumatoid arthritis. Deutsches Aerzteblatt 106:159-163

40. Naredo E, Iagnocco A (2012) Why use ultrasound in rheumatology? Rheumatology 51(suppl 7):vii1-vii1

41. Taggart A, Benson C, Kane D Ultrasound in rheumatology

42. Louthrenoo W (2014) An insight into rheumatology in Thailand. Nat Rev Rheumatol. doi:101038/nrrheum2014142

43. El Zorkany B, AlWahshi HA, Hammoudeh M, Al Emadi S, Benitha R, Al Awadhi A, Bouajina E, Laatar A, El Badawy S, Al Badi M (2013) Suboptimal management of rheumatoid arthritis in the Middle East and Africa: could the EULAR recommendations be the start of a solution? Clin Rheumatol 32(2):151-159

44. Moum B, Lundin KE (2014) Biosimilar medicines in inflammatory bowel disease. Tidsskr Nor Laegeforen Tidsskr Prakt Med Ny Raekke 134(8):819-820. doi:10.4045/tidsskr.14.0192

45. Radis C (2012) Rheumatoid arthritis: diagnosis and treatment with a particular emphasis on South Sudan. S Sudan Med J 5(4):85-88

46. Moon S, Jambert E, Childs M, von Schoen-Angerer T (2011) A 'winwin solution?': A critical analysis of tiered pricing to improve access to medicines in developing countries. Glob Health 7:39 
47. Freedman KB, Bernstein J (2002) Educational deficiencies in musculoskeletal medicine. J Bone Joint Surg 84(4):604-608

48. Oswald AE, Bell MJ, Snell L, Wiseman J (2008) The current state of musculoskeletal clinical skills teaching for preclerkship medical students. J Rheumatol 35(12):2419-2426

49. Thompson AE (2008) Improving undergraduate musculoskeletal education: a continuing challenge. J Rheumatol 35(12):2298-2299

50. Garneau KL, Iversen MD, Tsao H, Solomon DH (2011) Primary care physicians' perspectives towards managing rheumatoid arthritis: room for improvement. Arthritis Res Ther 13(6):R189

51. Harrold LR, Mazor KM, Negron A, Ogarek J, Firneno C, Yood RA (2013) Primary care providers' knowledge, beliefs and treatment practices for gout: results of a physician questionnaire. Rheumatology 52(9):1623-1629

52. Almeida C, Clarke B, O'Brien A, Hammond A, Ryan S, Kay L, Hewlett S (2006) Current provision of rheumatology education for undergraduate nursing, occupational therapy and physiotherapy students in the UK. Rheumatology 45(7):868-873

53. Hewlett S, Clarke B, O'Brien A, Hammond A, Ryan S, Kay L, Richards P, Almeida C (2008) Rheumatology education for undergraduate nursing, physiotherapy and occupational therapy students in the UK: standards, challenges and solutions. Rheumatology 47(7): $1025-1030$

54. Henrickson M (2011) Policy challenges for the pediatric rheumatology workforce: Part I. Education and economics. Pediatr rheumatol Online J 9:23. doi:10.1186/1546-0096-9-24

55. Li Z, Yang Y (2012) Rheumatology in China: challenges and development. Rheumatology 51(10):1733-1734

56. Lau C, Feng P (2007) Rheumatology without borders. Nat Clin Pract Rheumatol 3(6):305

57. Chipeta J, Njobvu P, McGill PE, Bucala R (2014) Progress made towards enhancement of rheumatology education and practice in Zambia: review of an ILAR-supported project. Clin Rheumatol 1-6

58. Berenbaum F (2014) The social (media) side to rheumatology. Nat Rev Rheumatol 10(5):314-318

59. Nair SC, Ibrahim H, Celentano DD (2013) Clinical trials in the Middle East and North Africa (MENA) region: grandstanding or grandeur? Contemp Clin Trials 36(2):704-710
60. Solovic I, Sester M, Gomez-Reino J, Rieder H, Ehlers S, Milburn H, Kampmann B, Hellmich B, Groves R, Schreiber S (2010) The risk of tuberculosis related to tumour necrosis factor antagonist therapies: a TBNET consensus statement. Eur Respir J 36(5):1185-1206

61. Registro Panamericano de Acontecimientos Adversos de terapias biológicas en enfermedades reumáticas. BIOBADAMERICA Website. https://biobadaser.ser.es/biobadamerica/. Accessed 5th November 2014

62. Sanchez-Riera L, Carnahan E, Vos T, Veerman L, Norman R, Lim S, Hoy D, Smith E, Wilson N, Nolla J (2014) The global burden attributable to low bone mineral density. Ann Rheum Dis 73(9): $1635-1645$

63. Chopra A (2013) The COPCORD world of musculoskeletal pain and arthritis. Rheumatology 52(11):1925-1928

64. eumusc.net. Musculoskeletal health key statistics, United Kingdom. http://www.eumusc.net/map/fs_pdf/FS_uk.pdf. Accessed 5th November 2014

65. eumusc.net. Musculoskeletal health key statistics, Ireland. http:// www.eumusc.net/map/fs_pdf/FS_ie.pdf. Accessed 5th November 2014

66. eumusc.net. Musculoskeletal health key statistics, France. http:// www.eumusc.net/map/fs_pdf/FS_fr.pdf. Accessed 5th November 2014

67. eumusc.net. Musculoskeletal health key statistics, Germany. http:// www.eumusc.net/map/fs_pdf/FS de.pdf. Accessed 5th November 2014

68. National Physician Survey, 2013. College of Family Physicians of Canada, Canadian Medical Association, Royal College of Physicians and Surgeons of Canada. http:// nationalphysiciansurvey.ca/wp-content/uploads/2013/10/NPS2013-Internal-Medicine-RRENr.pdf. Accessed 5th November 2014

69. Zhang F (2009) The China rheumatology workforce: a status report. Int J Rheum Dis 12(4):279-282

70. The World Bank, total population data by country. http://data. worldbank.org/indicator/SP.POP.TOTL. Accessed 5th November 2014 